\title{
Application of Rank Sequence Localization Algorithm based on Voronoi and Spearman in WSN
}

\author{
XuJianWei ${ }^{1}$ and WangDan ${ }^{2}$ \\ (Zhejiang Industry Polytechnic College Shaoxing Zhejiang 312000 China) \\ zhejiangxjw@163.com ${ }^{1}$,357437335@qq.com ${ }^{2}$
}

\begin{abstract}
Node localization technology is a key technology of WSN, and this paper proposes a rank sequence localization algorithm based on Voronoi and Spearman. First of al, the paper divides the localization space of WSN according to the Voronoi diagram, takes the polygon's points, center of gravity and the boundary intersection as virtuan anchor nodes, and builds the rank sequence list for virtual anchor nodes and anchor hodes. Afterwards, the paper calculates the Spearman's rank correlation coefficient of unknown nodes sequence and optimal sequence, and realizes the weighted estimate of unknown node location through coefficient normalization. The simulation results show that compared with ther algorithms, the proposed algorithm improves the sensor's node localization accuracy, reduges the atgolthm complexity, and has higher values.
\end{abstract}

Keywords: Rank Sequence; Locallation; WSN

\section{Introduction}

In wireless sensor netwo Rs (hereinafter referred to as WSN), node localization has been the research focus of senson technology. Whether the node localization is reasonable has largely determined the WSN caverage effects. However, the environmental factors of sensor nodes may affect the accurate localization to a certain extent. Among node localization, it is hard to localize the unknown nodes. How o define the unknown node location through anchor nodes has become a main direction of cunrent researches, and thus the localization technology has important theoretical and practical,significances when studying WSN [1].

For studies on WSN node self-localization, domestic and foreign scholars have made abundant research achievements [2]. According to the actually measured nodes' range or angle in the localization process, the paper divides the WSN localization into range-based localization or range-freelocalization [3]. The range-based localization mechanism is to determine the location of unknown nodes by measuring the actual range or angles of adjacent nodes, such as TOA-based localization, TDOA-based localization, AOA-based localization and RSSI-based localization [4, 5]. Range-free localization method needs not to measure the range between nodes, which only calculates the location of sensor nodes according to the network connectivity and beacon node information, such as the centroid algorithm [6], point-in-triangulation test algorithm [7], DV-HOP algorithm [8] and APIT algorithm [9].

To solve the current issues of rank sequence localization algorithm's high complexity and low localization accuracy in certain areas, based on Voronoi diagram, this paper proposes a sequence 
weighted WSN localization algorithm by introducing related concept of Spearman rank, uses the spatial division methods of Voronoi diagram and sequence localization ideas, relies on the import of virtual anchor nodes and the weighted estimate of optimal sequence, reduces the complexity of calculation while maintaining localization accuracy as high as possible, and effectively lowers network costs and energy consumption.

\section{Voronoi Diagram and Spearman's Rank Correlation Coefficient}

\subsection{Voronoi Diagram}

In Voronoi diagram, each basic graphic elements, being used to divide the space, is generally known as a point [10]. Voronoi diagram takes the planar point set $P$ as the point, which divides the plane into convex polygon-shaped Voronoi regions. In $P$, each point $P_{i}$ corresonds to one region $V_{i}$, so that the range between any points within $V_{i}$ is closer than the range between other points. Assumed the sensor point set in a two-dimensional plane $P=\left\{p_{1}, p_{2}, \ldots, \ldots, p_{n}\right\} \subset R^{m}$. The linear vertical plane bisecting $P_{i} \neq P_{j}$ and $p_{i} p_{j}$ divide the plane into two halves, and all scatter points' Voronoi regions constitute the scatter points set $P$ S Voronoi diagram $\mathrm{V}(P)$. By definition, $n$ scatter points divide the entire-wo-dimensional plane into $n$ units, and the Voronoi diagram is linearly proportional to the number, and thus it is highly efficiently to generate the Voronoi diagram. By using these reatures of Voronoi diagram, this paper divides the localization space and builds the virtualanchor nodes. As shown in Figure 1, the solid circle node refers to the virtual anchor nodes, whiteh can divide the two-dimensional region by building the Voronoi diagram.

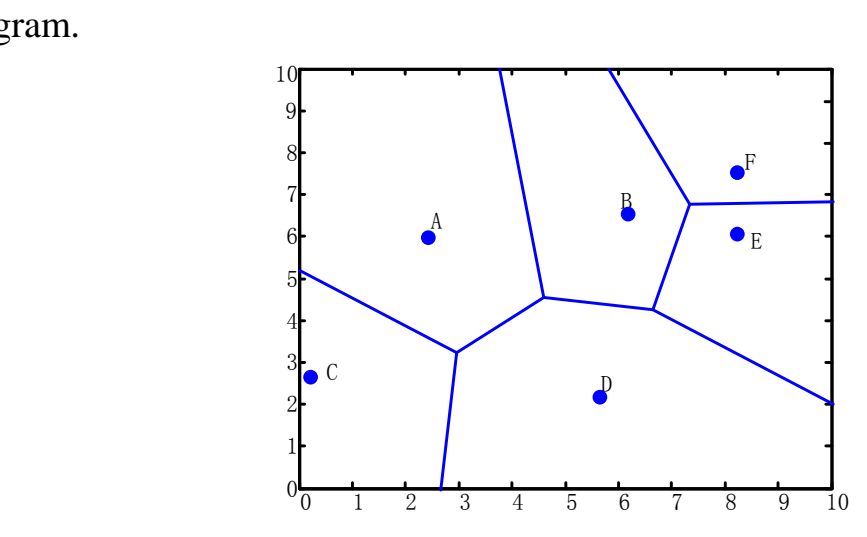

Figure 1. Construction of Two-Dimensional Voronoi Diagram

During the process of Voronoi division, the center of gravity's coordinate of the anchor nodes is the vertex of Voronoi polygon, and the coordinate of boundary intersection is its corresponding center of gravity. Voronoi polygon's plane center of gravity is calculated according to equations (1) and (2). Assumed that the plane center of gravity is $\left(P_{x}, P_{y}\right)$ and the polygon vertex is $\left\{\left(x_{i}, y_{i}\right) \mid 0 \leq i \leq p-1\right\}$. Where, $A=\sum_{i=0}^{p-1}\left(x_{i} y_{i+1}-x_{i+1} y_{i}\right)$, it is calculated as:

$$
P_{x}=\frac{1}{6 A} \sum_{i=0}^{p-1}\left(x_{i}+x_{i+1}\right)\left(x_{i} y_{i+1}-x_{i+1} y_{i}\right)
$$




$$
P_{y}=\frac{1}{6 A} \sum_{i=0}^{p-1}\left(y_{i}+y_{i+1}\right)\left(x_{i} y_{i+1}-x_{i+1} y_{i}\right)
$$

Side center of gravity is the key point of sides, and the point center of gravity is itself.

\subsection{Spearman's Rank Correlation Coefficient}

To better distinguish the correlation between two rank sequences, this paper mainly adopts Spearman's rank correlation coefficient, and concludes that Spearman's rank correlation coefficient indicator is more suitable for localization algorithm. According to the calculation of Spearman's rank correlation coefficient, in the given two localization sequence $U=\left\{u_{i}\right\}$ and $V=\left\{v_{i}\right\}, 1 \leq i \leq n$, two sequence's matching degree is determined by comparing all $\frac{n(n-1)}{2}$ ranks towards $\left(u_{i}, v_{i}\right)$ and $\left(u_{j}, v_{j}\right)$. If $u_{i}>u_{j} \Rightarrow v_{i}>v_{j}$ and $\backslash u_{i}<u_{j} \Rightarrow y_{i}>v_{j}$, the rank is believed to match or having the same sequence; If $u_{i}>y_{j} \Rightarrow v_{i}<v_{j}$ and $u_{i}<u_{j} \Rightarrow v_{i}>v_{j}$, the rank is believed to not match or having differen sequence. Finally, two localization sequence's Spearman's rank correlation coefficient ${ }^{[11]}$ is

$$
\tau=1-\frac{6}{n\left(n^{2}-1\right.} \sum_{i=1}^{n}\left(R_{i}-Q_{i}\right)
$$

Where, $R_{i}$ and $Q_{i}$ are respectively two different variables' rank in two localization sequences.

\section{Rank Sequence Localization Algorithm based on Voronoi and Spearman}

The idea of the proposed localization algorithm is: first uses Voronoi diagram to divide the anchor nodes in the localization space, constructs Voronoi polygons, builds the virtual anchor nodes through polygons center of gravity; builds a rank sequence list between virtual anchor nodes and actual anchor nodes, and finally determines the correlation coefficient between unknown node sequence and optimal sequence through this rank sequence list by using Spearman. Specific steps areas follows:

(1) Construct Voronoi space and determine the virtual anchor nodes. Number the anchor nodes with in the localized two-dimensional region, construct Voronoi polygon's vertex according to the Jocation of anchor nodes, number all polygons' nodes, and take the polygon's points, center of gravity and the coordinate of boundary intersection as the virtual anchor node. For the edge region, take the boundary intersection between Voronoi polygon and two-dimensional space as the virtual anchor node, which is beneficial for improving the localization accuracy of edge region.

(2) Build the virtual anchor node rank sequence. According to the virtual anchor nodes, determine the rank sequence of other nodes. Since coordinates of anchor nodes and virtual anchor nodes are known, the range can be calculated by using the range formula between two points, so 
as to determine the localization sequence of all virtual anchor nodes and get the localization sequence list.

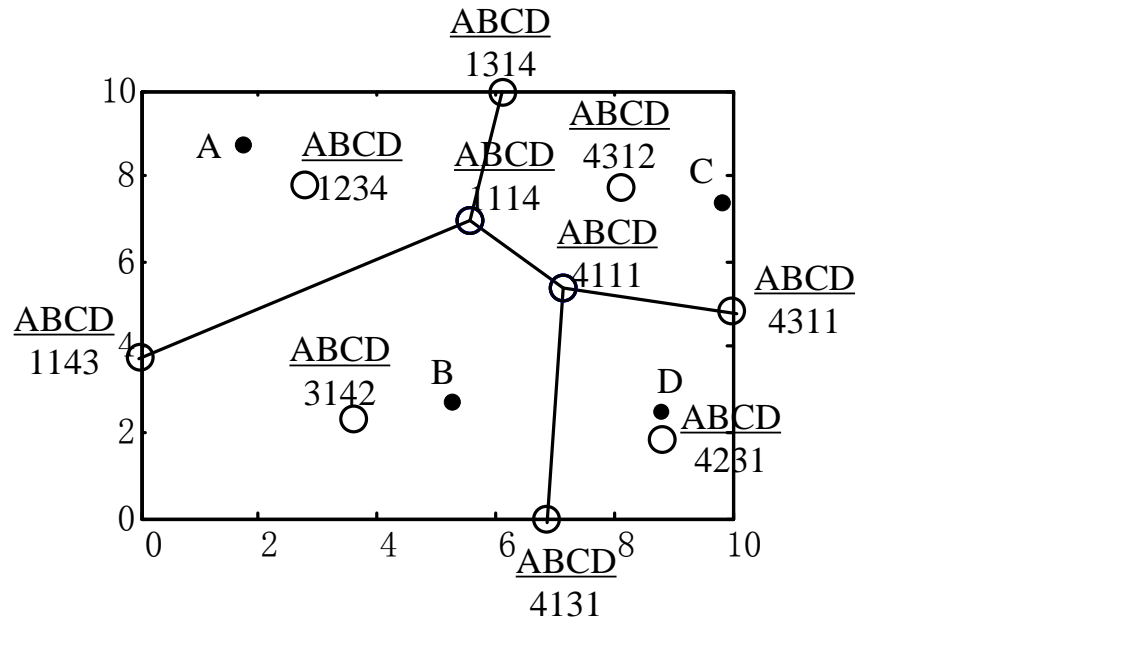

Figure 2. Virtual Beacon Node Localization Sequence

As shown in Figure 2, solid circles A, B, C, D are the beaconnodes, hollow circles represent the virtual beacon nodes, and the sequence beside each hollow circle is its localization sequence. Take the left-most virtual beacon nodesin Figure 2 as example, ABCD are the predefined sequence of beacon node number, 143 represents the localization sequence derived from the range formula, which means the virtual beacon node get closest to the nodes $\mathrm{A}$ and $\mathrm{B}$, followed by Node $\mathrm{D}$, and Node $\mathrm{C}$ is the farthest. Figare 2 shows the rank sequence of all virtual beacon nodes in the two-dimensional localization space. By Step 2, the rank sequence list of virtual beacon node can be constructed in any two-dimensional space.

(3) Use RSSI measurement to determine the range from each unknown sensor node to each beacon node, $\mathrm{O}$ as to determine its localization sequence. The whole network beacon nodes send packets to the surrounding nodes, and the unknown nodes divide the location sequence grade of sensor node to beacon node according to the obtained beacon node sequence.

(4) Use Spearnan to measure the correlation between different rank sequences. According to the given unknown node $X(x, y)$ 's rank sequence $U$, the proposed algorithm selects the optimal beacon node sequence from the rank list, and builds the virtual beacon node's optimal rank sequence list $T=\left\{T_{1}, T_{2}, \ldots, T_{M}\right\}$, which is contained in the optimal beacon node's Voronoi polygon. The conrelation at interval $[-1,1]$ can be normalized, transformed into the interval $[0,1]$ and take the Iransformed value as weight for the coordinates' weighted operation. The coordinate of node $X(\mathrm{x}, \mathrm{y})$ can be estimated as

$$
(\hat{x}, \hat{y})=\frac{1}{\sum_{i=1}^{M} \frac{1+\tau\left(T_{i}\right)}{2}} \sum_{i=1}^{M}\left(\frac{1+\tau\left(T_{i}\right)}{2} V B_{i}\right)
$$

Where, $V B_{i}$ is the coordinate of virtual anchor node corresponding to the $i$-th sequence in the optimal rank sequence list $T$. 


\section{Simulation Experiment}

\subsection{Simulation Environment}

This paper uses Matlab simulation software to make simulation analysis of and assess the proposed algorithm's performance, and selects 200 nodes to randomly distribute in a $10 \mathrm{~m} \times 10 \mathrm{~m}$ square area. The number of anchor nodes is respectively of 4, 12, 36, 108, the node communication radius is $2 \mathrm{~m}$, and all anchor nodes are located in the wireless range of unknown nodes.

In order to describe the performance of the proposed algorithm, this paper compares it with the sequence localization algorithm and sequence three-point orthocentric algorithm from the aspects of localization error and calculation time. The simulation localization error $P_{e}$ is definedas:

$$
P_{e}=\frac{\mid \text { Calculatedlocation }- \text { actuallocation } \mid}{\text { wirlesscommunicationradius }} \times 100 \%
$$

\subsection{Results and Analysis}

4.2.1. Comparison of Localization Accuracy: Figure-3 shows the localization errors of three algorithms. According to Figure 4, although the number of anchor nodes varies, the proposed algorithm increases by about $8 \%$ as compared with the sequence three-point orthocentric algorithm and sequence localization algorithm. Due the introduction of virtual boundary anchor nodes and Spearman, the proposed algorithm still remains low localization error when there are a small number of anchor nodes.

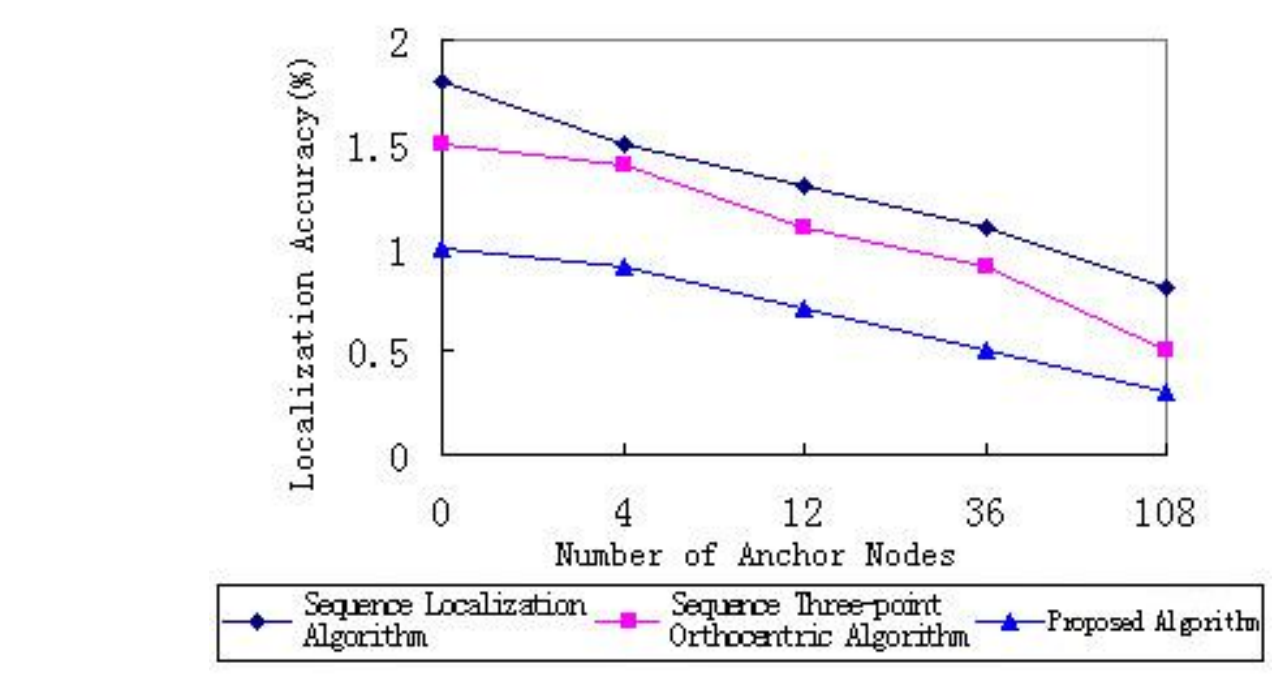

Figure 3. Comparison of Different Algorithms' Localization Error

4.2.2. Comparison of Localization Time: Figure 4 respectively shows the time comparison of three algorithms. According to Figure 5, with the increase of anchor nodes, this algorithm time has been improved, and the sequence localization algorithm in literature [5] and the sequence three-point orthocentric algorithm in literature [6] have the same complexity. The spatial and temporal complexity are respectively of $O\left(\mathrm{n}^{6}\right)$ and $O\left(\mathrm{n}^{5}\right)$. The proposed algorithm's spatial and 
temporal complexity for construction of Voronoi diagram in two-dimensional space is $O\left(\mathrm{~m}^{2}\right)$, the spatial and temporal complexity for construction of virtual anchor nodes is $O\left(\mathrm{n}^{3}\right)$, and the spatial and temporal complexity for calculation of Spearman's correlation coefficients is $O\left(\mathrm{~m}^{2}\right)$. Where, $\mathrm{m}<\mathrm{n}$, so the proposed algorithm's spatial and temporal complexity is $O\left(\mathrm{n}^{3}\right)$, which has been reduced as compared with other sequence localization algorithm's complexity.

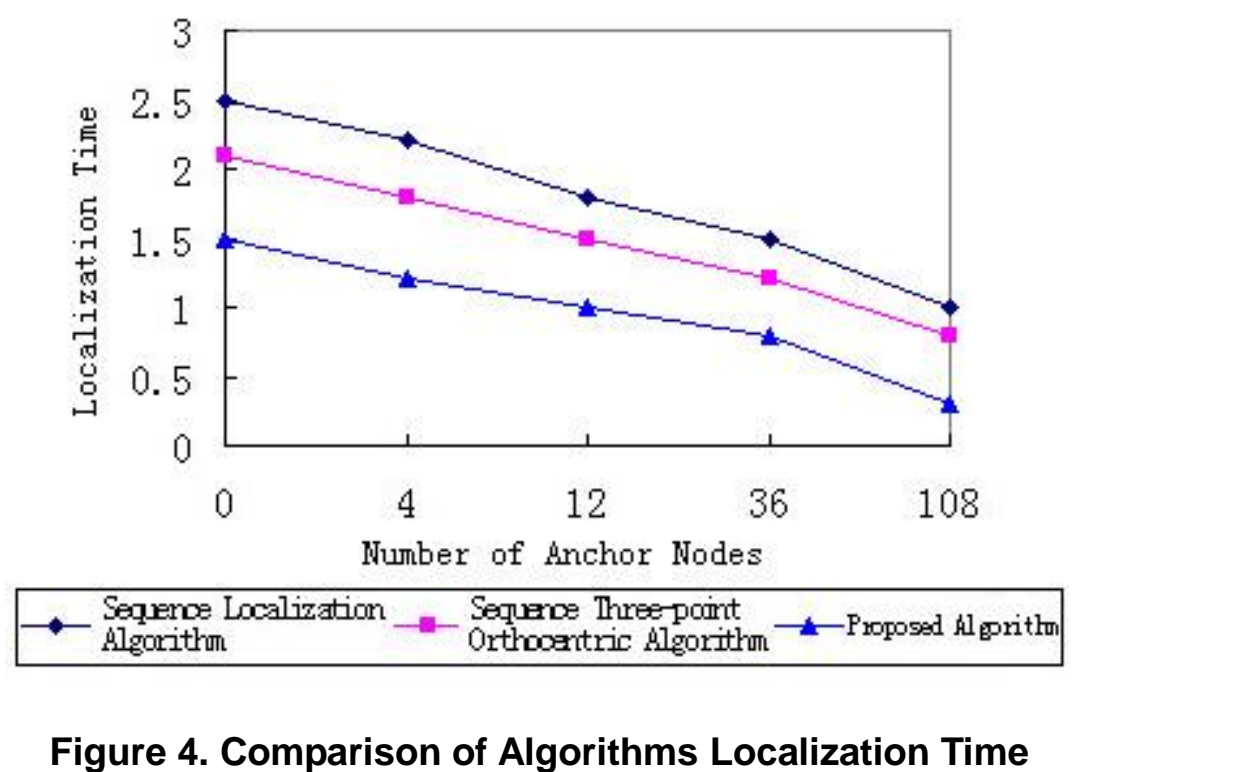

\section{Conclusion}

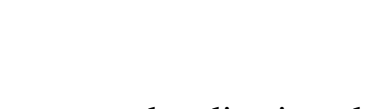

To solve the issue of rank sequence localzation algorithm's high complexity and low boundary region localization accuracy, thin paper proposes a rank sequence weighted wireless sensor node localization algorithm First of all, based on Voronoi diagram's division of localization space, this paper takes the polyon s points, center of gravity and the boundary intersection as virtual anchor nodes, and builds the rank sequence list from virtual anchor nodes to anchor nodes. Finally, the proposed algorithm calculates the Spearman's rank correlation coefficient of unknown nodes sequence and optimal sequence, and realizes the weighted estimate of unknown node location through coefficien normalization. The simulation results show when ensuring high localization accuracy, the proposed algorithm reduces the algorithm complexity and produces no extra network costs and energy consumption.

\section{References}

[1] Z. Ma, Y. Sun and T. Mei, "Summary for wireless sensor network [J]", Journal of China Institute of Communications, vol. 25, no. 4, (2004), pp. 114-124.

[2] Queyan, Zhao Chong Chong, Dai Gui Lan, "Research on Localizaiton Technology for Wireless Sensor Networks [J]", Computer Science, vol. 35, no. 5, (2008), pp. 47-50.

[3] Diao Ying Fei, "Localization Problem in Wireless Sensor Network [D]”, ShangDong University, (2013). 
[4] Tan Zhi and Zhang Hui, "A Modified Mobile Location Algorithm Based on RSSI [J]", Journal of Beijing University of Posts and Telecommunications, vol. 36, no. 3, (2013), pp. 88-91.

[5] Li Wen Cheng and Zhang Lei, "Research on Weighted Centroid Location Algorithm for wireless Sensor Network [J]. Computer Simulation, vol. 30, no. 2, (2013), pp. 191-194.

[6] V. Martusevcius and E. Kazanavicius, "Self-localization system for wireless sensor network [J]", Elektronika Ir Elektrotechnika, vol. 7, no. 103, (2010), pp. 17-20.

[7] N. Bulusu, "GPS-less Low Cost Outdoor Localization for Very Samll Devices [J]", IEEE Personal Communications Magazine, vol. 7, no. 5, (2000), pp. 28-34.

[8] D. Nicolescu , “Ad Hoc Positioning Systems[C]/Proc.of IEEE GLOBECOM’01.[S.1]:IEEE Press,2001

[9] T. He and H. Chengdu, "Range-free Localization Schemes for Large-scale Sensor Networks[C]//Porc of the $9^{\text {th }}$ Annual International conference on Mobile Computing and Networking, New York, USA:[s.n], (2003).

[10] Zhou Pei De, "Calculation of geometric algorithm design and analysis [M]”, Beijing: Tsinghua University press, (1941), pp. 126-139.

[11] Wang Kai Jun, "Spearman Rank Correlation Method Based on Trend Rank [J]”, Journal of Fujian Normal University(Natural Science Edition), vol. 26, no. 1, (2010), pp. 38-41.

\section{Authors}

Xu Jianwei: (1980- ), Male, Master's Degree, Research Direction: intelligent control, embedded system technology, detection device ând instrumentation.

Wang Dan: (1978- ), Female Master"s Begree, Research Direction: mechanical and electrical control and automation, dynamic testing and fault diagnosis of mechanical system

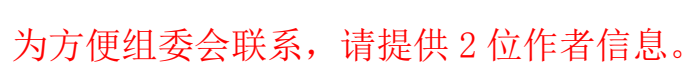

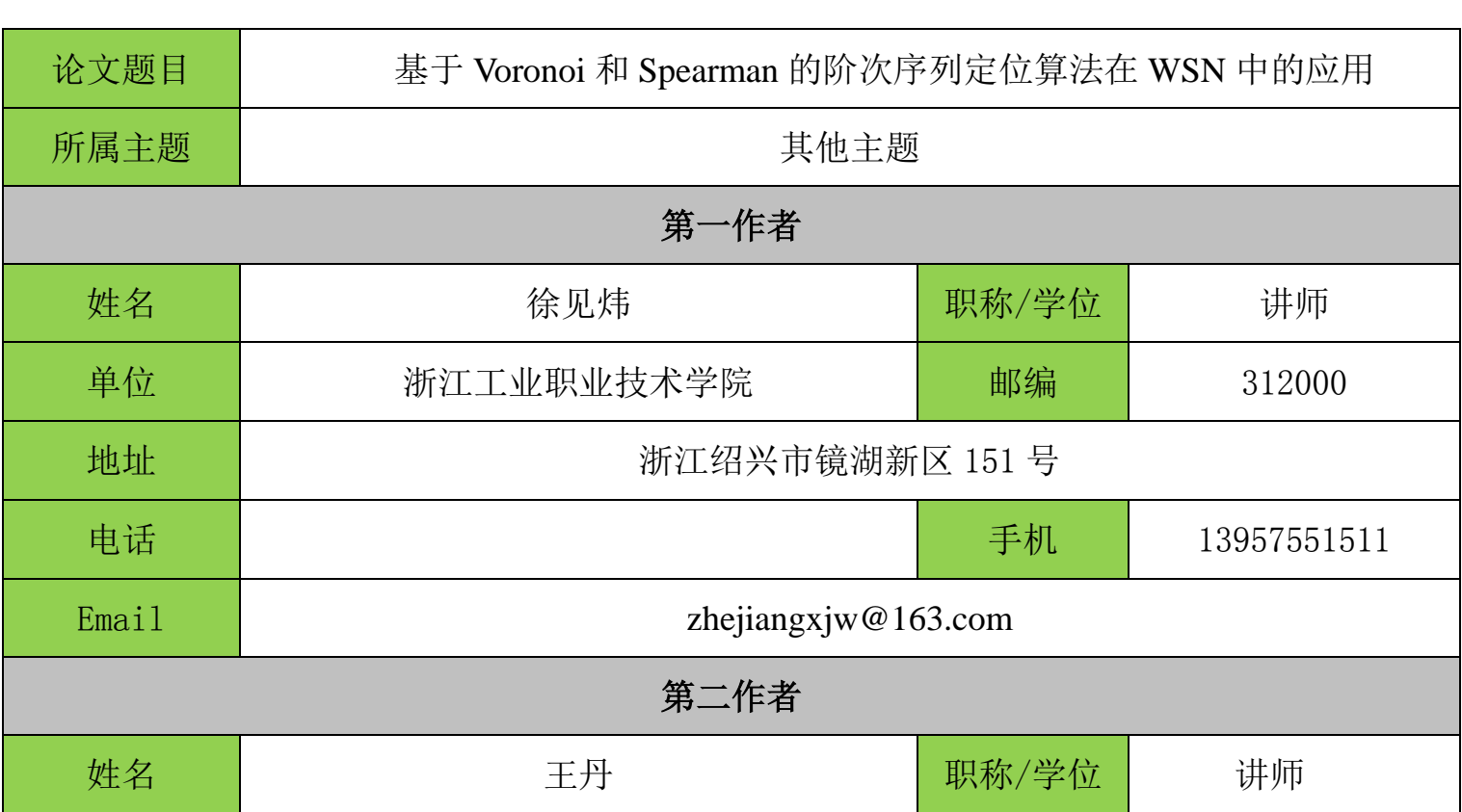


International Journal of Smart Home

Vol. 8, No. 6 (2014)

\begin{tabular}{|c|c|c|c|}
\hline 单位 & 浙江工业职业技术学院 & 邮编 & 312000 \\
\hline 地址 & \multicolumn{3}{|c|}{ 浙江绍兴市镜湖新区 151 号 } \\
\hline 电话 & & 手机 & 13957551522 \\
\hline Email & \multicolumn{3}{|c|}{ 357437335@qq.com } \\
\hline
\end{tabular}

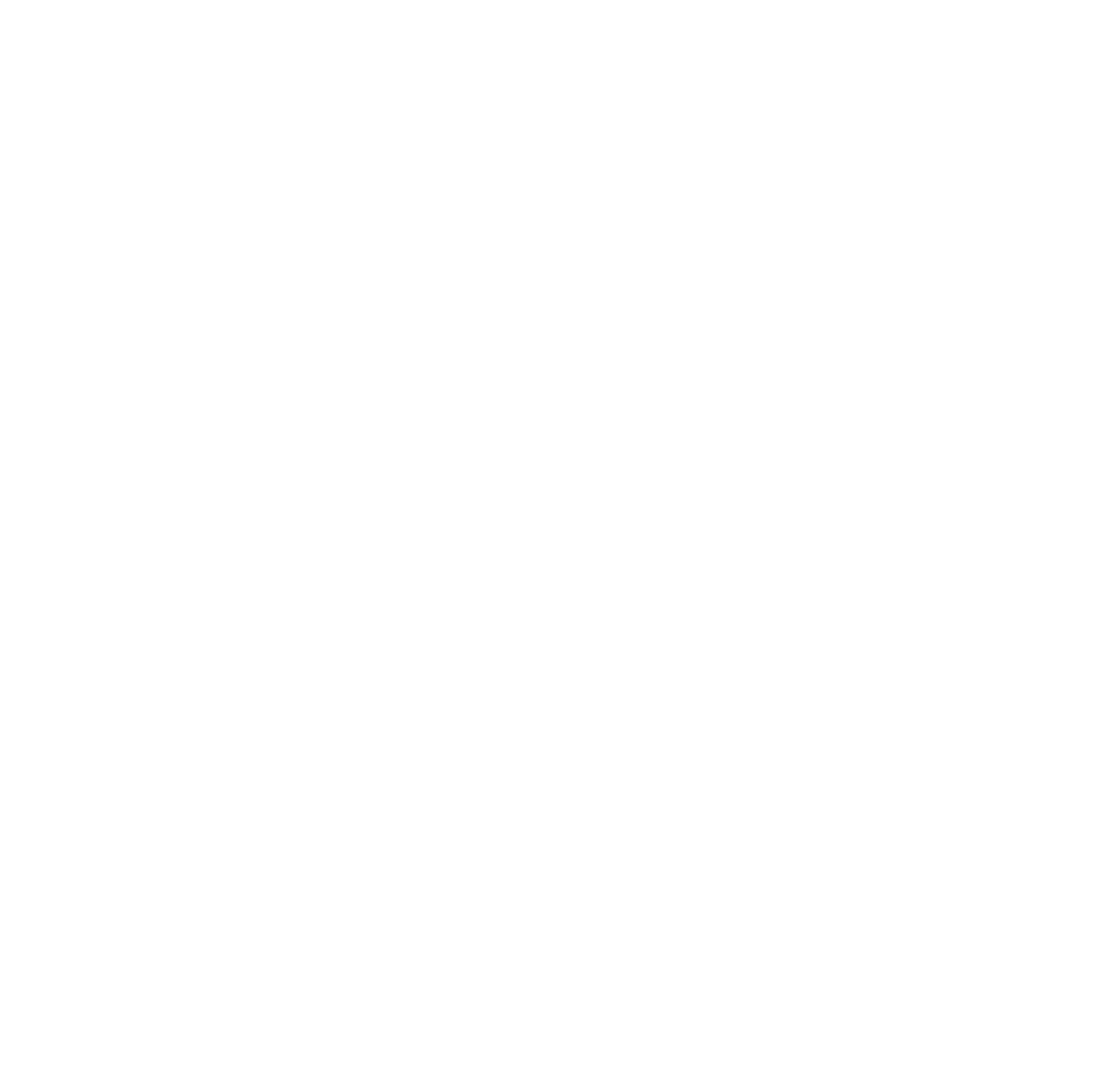

